\title{
Estudio comparativo de la composición florística, diversidad y productividad de vegas o mallines de Tierra del Fuego, Chile
}

\author{
ERWIN DOMÍNGUEZ1 $\ \&$ \& CLAUDIO PÉREZ1 \\ 1. https://orcid.org/0000-0002-1907-8399
}

\section{OPEN ACCESS RESUMEN}

Recibido:

21/09/2021

\section{Revisado:}

09/12/2021

\section{Aceptado:}

$14 / 12 / 2021$

\section{Publicado en línea:}

$31 / 12 / 2021$

Editor:

Dr. Americo Montiel San Martin.

ISSN 0718-686X

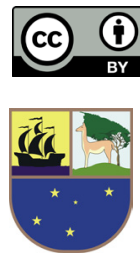




\section{Comparative study of the floristic composition, diversity and productivity of the wet meadows or mallines of Tierra del Fuego, Chile}

\begin{abstract}
CÓMO CITAR:
Dominguez et al. (2021).

Estudio comparativo de la

composición florística, diversidad y productividad de vegas o mallines de

Tierra del Fuego, Chile.

Anales Instituto de la Patagonia, https://doi.org/10.22352/

AIP202149016

Contribución de los autores:

ED: Adquisición, análisis, interpretación de los datos florísticos

y elaboración del manuscrito.

CP: Adquisición e interpretación de los datos agronómicos.

Declaración de interéses:

Los autores declaran no tener conflicto de intereses.

Financiamiento: Sin financiamiento.
\end{abstract}

homogéneas en su composición de especies, ni en su estructura, estas muestran evidencias de cambios en su diversidad florística, debido a los impactos generados por la intensidad del pastoreo, el cual se refleja en producción de forraje, fluctuando entre 408 a $4.055 \mathrm{~kg} \mathrm{MS} \mathrm{ha}^{-1}$.

Palabras claves: Humedal, Diversidad florística, Patagonia.

\section{ABSTRACT}

Wet meadow or mallines from a functional and productive point of view, are a fundamental component in the livestock systems of Patagonia, as they are the main source of forage. With the working hypothesis that, the generation of knowledge about the floristic diversity and productivity of the different types of plains present in Magallanes, will promote a sustainable livestock management of these, which, in turn, will guarantee the conservation of the functions and wet meadow ecosystem services. Between January 2019 and May 2020, a study was carried out in six estancias on the Island of Tierra del Fuego, Chile, to describe the taxonomic components, compare the diversity of plant species, evaluate the floristic similarity, along with analyzing the temporal distribution from plant biomass to the exclusion of herbivory that characterize these ecosystems. A total of 53 taxa were recorded, distributed in 16 families (Poaceae, Rosaceae, Cyperaceae, Apiaceae and Asteraceae, mainly) and 40 genera, of which 40 species are native and 11 introduced to the plains. The wet meadows analyzed presented specific richness values and diversity indices lower than those found in Patagonia Argentina. The floristic composition and the attributes of the vegetation showed patterns linked to the water content of the soil, observing a negative relationship between the water table and the values of richness and diversity indices. Regarding vegetation cover, the humid sectors presented higher percentages of vegetal cover than those of the semi-humid sectors. Finally, it was determined that the wet meadows are not homogeneous in their composition of species, nor in their structure, they show evidence of changes in their floristic diversity, due to the impacts generated by the intensity of grazing, which is reflected in forage production, fluctuating between 408 to $4,055 \mathrm{~kg}$ DM ha- ${ }^{1}$.

Key words: Wetland, Floristic diversity, Patagonia. 


\section{INTRODUCCIÓN}

Las vegas o mallines constituyen humedales altamente productivos, que aportan diversos servicios ecosistémicos. Uno de ello es ser fuente de agua y alimento, especialmente para la ganadería ovina, y fauna silvestre, por su aporte de forraje de calidad y en abundancia. Estos humedales en la Patagonia se caracterizan por constituir áreas saturadas de agua, en forma permanente o temporal durante el año, las que provienen del aporte de agua subterránea o estacional, generada por el escurrimiento superficial de los deshielos o de las precipitaciones estacionales (Pisano 1977; Filipová et al. 2013). Siendo éste el factor dominante que determina la naturaleza del desarrollo de un suelo abundante en materia orgánica y de una vegetación herbácea típica, formada por juncáceas, ciperáceas y gramíneas. Las vegas en la Región de Magallanes, cubren una superficie aproximada de 200.000 hectáreas (SAG 2004), y se desarrollan en depresiones entre morrenas o fondos de valles.

Las vegas constituyen ecosistemas altamente productivos que aportan de agua y alimento, a la ganadería, principalmente forraje de calidad, con alto valor nutricional. Sin embargo, debido a la sobrecarga animal a que son sometidos estos humedales por la actividad ganadera, se ha producido una degradación de estos ambientes con la pérdida de diversidad florística. No obstante, algunos estudios en el hemisferio norte han mostrado efectos beneficiosos sobre la riqueza de especies vegetales al realizar un pastoreo racional (Dupré $E$ Diekmann, 2001; Pykälä, 2005). Por otra parte, la mayoría de los estudios en Patagonia especialmente en Argentina, sobre las vegas o mallines se han focalizado en los aspectos geológicos, edáficos, sedimentológicos, topográficos, hídricos, mapeos y procesamiento de imágenes satelitales y sobre la distribución espacial de las comunidades vegetales (Gandullo \& Schmid, 2001; Gaitán et al. 2011; Mazzoni E Rabassa; 2013; Vázquez et al. 2013; Gaitán et al. 2015; Grima et al. 2015; Andreazzini et al. 2020). Los dos únicos trabajos en Magallanes a una escala regional sobre la variabilidad de tipos de suelo y clasificación de vegetación en vegas, corresponde a los estudios realizados por Filipová et al. 2010 y Filipová et al. 2013, y un reciente estudio de caso sobre la caracterización de la composición y estructura florística en las geoformas asociadas a una vega, acotado a una estancia de uso ganadero (Domínguez $\varepsilon$ Ivelic-Sáez, 2020). Bajo este escenario, se requiere generar más conocimiento acerca de los distintos tipos de vegas presentes en Magallanes y como estas difieren en diversidad y productividad, principalmente en sectores con tendencia al aumento en la intensificación de uso de la actividad ganadera. Esta información ayudará a propiciar un manejo ganadero sustentable, garantizando la conservación de las funciones y servicios ecosistémicos. Por lo anterior, los objetivos del presente estudio son: 1) describir los componentes taxonómicos que caracterizan a las vegas de Tierra del Fuego, 2) comparar la biodiversidad de especies entre ellas, 3) evaluar la similitud o diferencias florísticas, junto con identificar las especies que estructuran estos humedales y 4) analizar la distribución temporal de la biomasa vegetal a la exclusión de la herbivoría, entre los distintos tipos de vegas estudiadas.

\section{METODOLOGÍA}

Sitio de estudio

Entre enero de 2019 y mayo 2020, se evaluaron seis vegas en las estancias: Ida Irene ( $52^{\circ} 39^{\prime} 44.15^{\prime \prime}$ S., $69^{\circ} 16^{\prime} 21.70^{\prime \prime}$ O.), Punta Catalina ( $52^{\circ} 38^{\prime} 57.65^{\prime \prime}$ S., $68^{\circ} 45^{\prime} 0.26^{\prime \prime}$ O.), San Miguel ( $52^{\circ} 51^{\prime} 49.83^{\prime \prime}$ S., $69^{\circ} 20^{\prime} 23.58^{\prime \prime O}$.), Millaray ( $53^{\circ} 2^{\prime} 28.99^{\prime \prime S}$., $68^{\circ} 53^{\prime} 20.23^{\prime \prime O}$.), La Frontera 


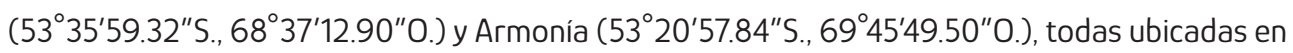
la Isla de Tierra del Fuego (Figura 1). Las vegas estudiadas Ida Irene, Punta Catalina, San Miguel, Millaray, se encuentran dentro del distrito agroclimático de Cerro Sombre (CS). Este distrito se caracteriza por presentar entre 200 a 250 días de crecimiento, 7 meses de déficit hídrico, 294 $\mathrm{mm}$ de precipitación anual y $866 \mathrm{~mm}$ de evapotranspiración potencial anual. Por otra parte, la vega Armonía se encuentra dentro del distrito agroclimático de Porvenir (PV) caracterizada por presentar entre 200 a 250 días de crecimiento, 6 meses de déficit hídrico, 388 mm de precipitación anual y $793 \mathrm{~mm}$ de evapotranspiración potencial anual y finalmente la vega La Frontera se encuentra dentro del distrito agroclimático de Bahía Inútil (BI) caracterizada por presentar entre 150 a 199 días de crecimiento, 6 meses de déficit hídrico, 347 mm de precipitación anual y 739 mm de evapotranspiración potencial anual (Domínguez et al. 2019).

\section{Diseño de muestreo}

Se instalaron 9 jaulas de exclusión de $0,72 \mathrm{~m}^{2}$ ubicadas al azar, en el centro de cada vega, donde se registró la riqueza y abundancia de las especies vegetales, utilizando la escala modificada de Braun Blanquet (Van der Maarel, 2007). Además, en cada una de estas 9 jaulas de exclusión, fue cosechada la biomasa aérea durante la estación de crecimiento, desde mayo de 2019 a febrero de 2020, utilizando un aro metálico de $0,1 \mathrm{~m}^{2}$. Las muestras fueron secadas en estufa de aire forzado (Memmert UF450) a 60oC por 48 hrs, hasta obtener el peso constante. Los trabajos de identificación de plantas se realizaron con claves de acuerdo a la Flora Patagónica de INTA (Correa, 1969, 1971, 1978, 1984, 1985, 1988, 1999). La nomenclatura de las especies, su hábito y origen, se basó en el Catálogo de Plantas Vasculares de Chile (Rodríguez y Marticorena, 2019).

\section{Análisis estadístico}

Para cada unidad experimental (vega), se calculó la riqueza específica (S), la cobertura vegetal (\%), la diversidad mediante índice de Diversidad de Shannon-Weaver ( $\left.\mathrm{H}^{\prime}\right)$, esto con el objeto de comparar las distintas relaciones especie-abundancia entre las vegas estudiadas. También se estimó el índice de Diversidad de Dominancia (D) e inverso de Simpson (1-D), junto con el índice de Equitatividad de Pielou. Este índice reduce el efecto del submuestreo de especies $y$, por lo tanto, permite una mejor comparación entre comunidades con alta dominancia de unas pocas especies. Con los datos obtenidos se realizó la prueba de Kruskal-Wallis con la corrección de Bonferroni, para detectar diferencias entre las vegas, debido a que los datos no mostraron distribución normal (prueba de Shapiro-Wilk). Los datos de riqueza y cobertura de especies de las vegas estudiadas, fueron tabulados, creando una matriz recíproca. Con esta información se aplicaron índices de distancia de Bray-Curtis y análisis de componentes principales (PCA) con el fin de establecer gradientes ecológicos que estructuran la composición de las vegas estudiadas. Para determinar la contribución de las especies en la estructuración de la comunidad de plantas, se realizó un análisis de porcentaje de similitud (SIMPER) (Clarke, 1993), de modo de indicar cuantitativamente qué especies vegetales explican las diferencias entre grupos. Los análisis, tanto univariados como multivariados, se realizaron con el programa PAST (Palaeontological Statistics) v 4.0 (Hammer et al. 2001). Con los datos de forraje obtenidos, se realizó la prueba de Kruskal-Wallis con la corrección de Bonferroni, para detectar diferencias entre las vegas, debido a que los datos no mostraron distribución normal (prueba de Shapiro-Wilk). Todos los análisis estadísticos se realizaron con el programa INFOSTAT (Di Rienzo et al. 2011). 
Figura. 1.

Mapa de localización de las vegas estudiadas en la Tierra del Fuego. Nombre de los humedales: 1) Ida Irene, 2) Punta Catalina, 3) San Miguel, 4) Millaray, 5) La Frontera y, 6) Armonía.

Figura 2. Jaulas de exclusión de $0,72 \mathrm{~m}^{2}$ instaladas en cada vega, con el fin de evitar la herbivoría y el pisoteo por parte del ganado ovino. A) Superficie excluida en la jaula de exclusión y B) Corte de $0,1 \mathrm{~m}^{2}$.
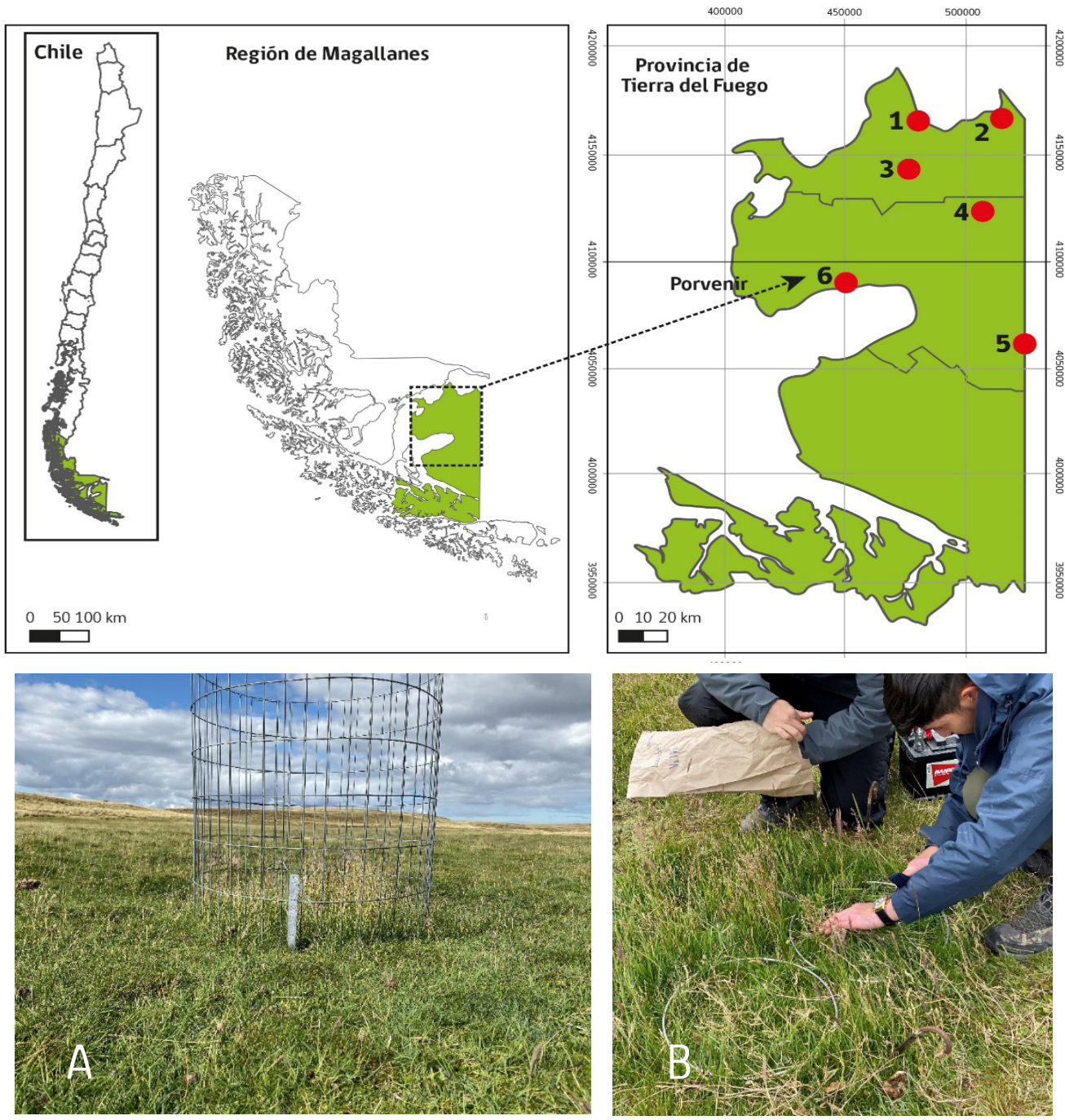

\section{RESULTADOS}

Se registraron un total de 53 taxones para las vegas, distribuidos en 16 familias y 40 géneros, de las cuales 40 especies son nativas y 11 introducidas. Tres especímenes no pudieron ser identificados a nivel específico por no presentar estructuras reproductivas, y solo pudieron ser determinadas a nivel de género (Poa sp. y Ranunculus sp.) y el tercer espécimen a nivel de división (Briophyta). Las familias mejor representadas en relación a la frecuencia absoluta fueron: Poaceae, Rosaceae, Cyperaceae, Apiaceae y Asteraceae (Figura 3). La vega con mayor riqueza de especies fue La Frontera $(10,56 \pm 1,59)$, mientras que Armonía presentó la menor riqueza de especies $(4,22 \pm 1,3)$, siendo ambas diferentes significativamente entre sí y con respecto el resto a las otras vegas estudiadas. La cobertura promedio para las vegas fluctuó entre un 96 a 100\%, siendo significativamente distinta la vega Ida Irene, del resto. Los índices de Dominancia (D) y Simpson (1-D) muestran que Punta Catalina es la vega más diversa. Esto se ajusta a lo resultados obtenidos con el índice de diversidad de Shannon-Wiener $\left(H^{\prime}=1,67 \pm 0,28\right)$. La Equitatividad obtenida 
Figura 3.

Frecuencia absoluta de familias identificadas en las vegas en Tierra del Fuego.
Figura 4. Ordenación mediante un Análisis de Componentes Principales (PCA) para los 54 inventarios realizados en seis vegas en Tierra del Fuego. Muestra

la formación de 4 grupos: Grupo 1: Ida Irene; Grupo 2: Punta Catalina, San Miguel y Millaray, Grupo 3: La Frontera y Grupo 4: Armonía

Letras minúsculas distintas representan diferencias significativas ( $p<$ 0,05). Ar: Armonía,

II: Ida Irene, LF: La Frontera, MI: Millaray, PC: Punta Catalina y SM: San Miguel.

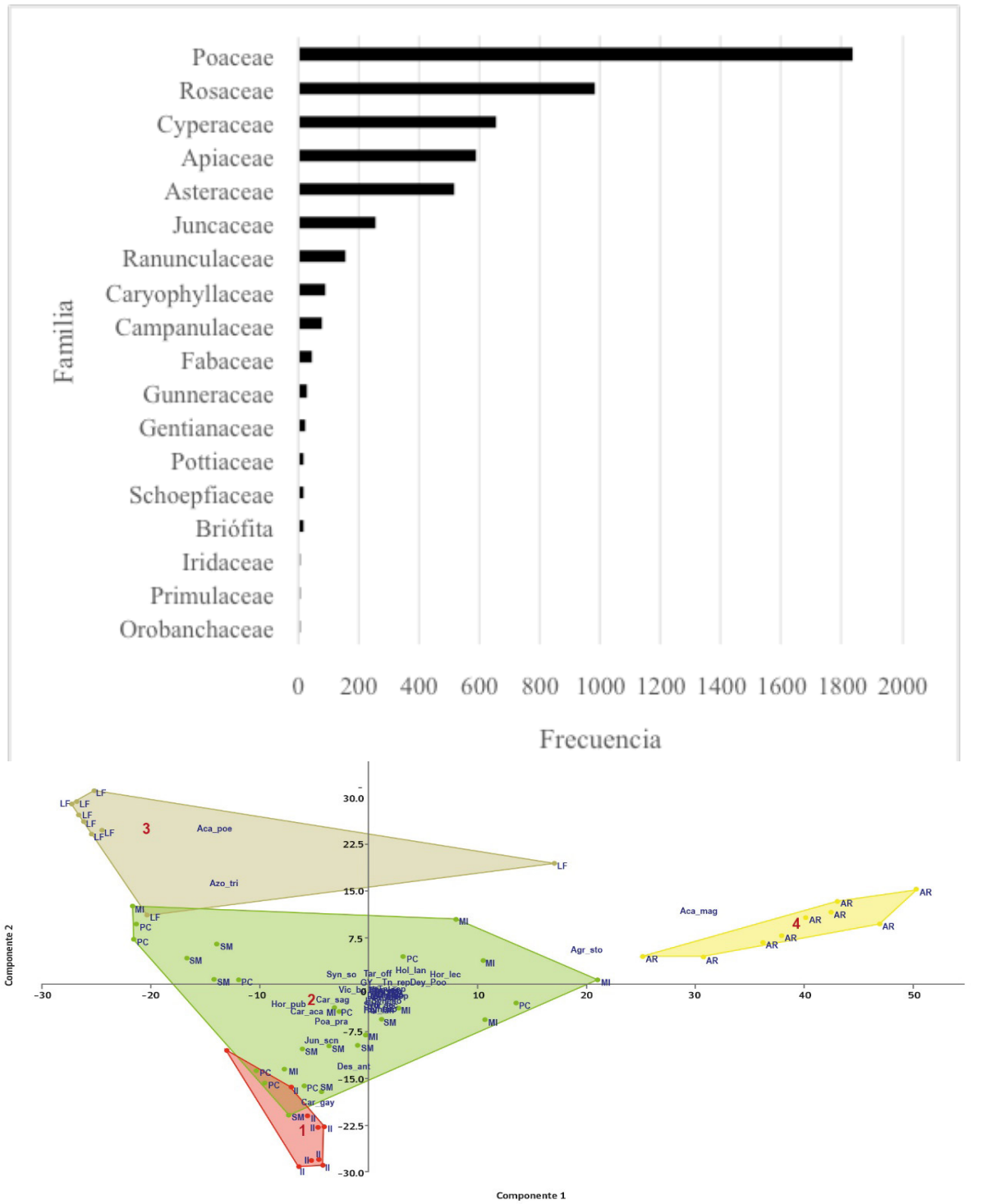

Tabla 1. Promedio ( $\pm \mathrm{DE}$ ) de los parámetros comunitarios de biodiversidad: riqueza de especies (S), cobertura vegetal (\%), índice de dominancia (D), índice de Simpson (1-D) e índice de Shannon-Wiener $\left(\mathrm{H}^{\prime}\right)$ para las vegas estudiadas.

\begin{tabular}{lcccccc}
\hline Parámetros comunitarios & AR & $\|$ & LF & MI & PC & SM \\
\hline Riqueza de especies (S) & $4,22 \pm 1,3^{\mathrm{a}}$ & $8,22 \pm 2,59^{\mathrm{bc}}$ & $10,56 \pm 1,59^{\mathrm{c}}$ & $9,11 \pm 3,26^{\mathrm{bc}}$ & $7,67 \pm 2,12^{\mathrm{b}}$ & $8 \pm 1,14^{\mathrm{b}}$ \\
Cobertura Vegetal (\%) & $100 \pm 0,0^{\mathrm{b}}$ & $94,89 \pm 5,04^{\mathrm{a}}$ & $98,67 \pm 1,12^{\mathrm{a}}$ & $97,22 \pm 3,96^{\mathrm{ab}}$ & $100 \pm 0,0^{\mathrm{b}}$ & $98,89 \pm 0,93^{\mathrm{a}}$ \\
Dominancia (D) & $0,42 \pm 0,07^{\mathrm{b}}$ & $0,23 \pm 0,07^{\mathrm{a}}$ & $0,28 \pm 0,04^{\mathrm{a}}$ & $0,26 \pm 0,09^{\mathrm{a}}$ & $0,23 \pm 0,07^{\mathrm{a}}$ & $0,25 \pm 0,04^{\mathrm{a}}$ \\
Simpson (1-D) & $0,58 \pm 0,07^{\mathrm{a}}$ & $0,77 \pm 0,07^{\mathrm{b}}$ & $0,72 \pm 0,04^{\mathrm{b}}$ & $0,74 \pm 0,09^{\mathrm{b}}$ & $0,77 \pm 0,07^{\mathrm{b}}$ & $0,75 \pm 0,04^{\mathrm{b}}$ \\
Shannon-Wiener (H') & $1,04 \pm 0,2^{\mathrm{a}}$ & $1,58 \pm 0,3^{\mathrm{b}}$ & $1,6 \pm 0,18^{\mathrm{b}}$ & $1,64 \pm 0,33^{\mathrm{b}}$ & $1,67 \pm 0,28^{\mathrm{b}}$ & $1,6 \pm 0,13^{\mathrm{b}}$ \\
Equitatividad (J) & $0,75 \pm 0,08^{\mathrm{b}}$ & $0,83 \pm 0,1^{\mathrm{c}}$ & $0,68 \pm 0,05^{\mathrm{a}}$ & $0,76 \pm 0,07^{\mathrm{bbc}}$ & $0,84 \pm 0,07^{\mathrm{c}}$ & $0,8 \pm 0,08^{\mathrm{bc}}$ \\
\hline
\end{tabular}


fue $0,84 \pm 0,07$, lo cual indica que la mayor parte de especies registradas en la vega Punta Catalina, comparten abundancias similares. Por otra parte, la vega con menor diversidad, fue la vega Armonía $\left(H^{\prime}=1,04 \pm 0,2\right)$, siendo esta la única que se diferencia en forma significativa respecto a la riqueza de especie (S) y el índice de Shannon-Wiener $\left(H^{\prime}\right)$ (Tabla 1).

La figura 3 muestra la segregación de las especies en el plano formado por los dos primeros componentes principales, que suman una varianza acumulada de $91 \%$. El componente 1 explica un 56\% de variación y el componente 2 explica el 35\% de variación. La mayoría de las especies se ubican en la intersección de los ejes, sin mayor diferenciación. No obstante, el análisis separó claramente las muestras en cuatro grupos. Observándose, que 7 especies se segregaron tanto en el primero como en el segundo componente.

El componente 1, representan un gradiente de pastoreo que aumenta de izquierda a derecha (Fig. 3). A la izquierda se aprecia a Azorella trifurcata maleza indicadora de mala condición y a la derecha Acaena magellanica y Agrostis stolonifera como indicadoras de buena condición (Figura 3). El componente 2 representa el nivel freático del suelo, siendo las especies tolerantes al anegamiento Deschampsia antarctica y Carex gayana, especialmente, en la vega Ida Irene. La ordenación muestra como una zona intermedia que incluye a las vegas: San Miguel, Punta Catalina y Millaray, como un área de transición, mientras que la vega La Frontera representa la comunidad más seca, siendo Acaena poeppigiana y Azorella trifurcata, las especies más correlacionadas.

Las especies más importantes que estructuran las vegas fueron: Carex gayana (21,7\%), Deschampsia antarctica (14,8\%), Hordeum pubiflorum (13,8\%) en el grupo1; Hordeum pubiflorum (21,8\%9, Carex acaulis (12,3\%), Azorella trifurcata (11,3\%), Taraxacum officinale (10\%) en el grupo 2; Acaena poeppigiana (32,9\%), Azorella trifurcata (26,9\%), Hordeum pubiflorum (13,7\%) en el grupo 3 y Acaena magellanica (40,4\%), Agrostis stolonifera (23,4\%), Hordeum pubiflorum (7,89\%) en el grupo 4 (Tabla 2 ).

La biomasa obtenida representa la producción acumulada (KgMS ha-1) para seis vegas, durante 7 meses de evaluación entre los años 2019 y 2020. Estas entregaron tres niveles de producción: bajo, moderado y alto (Fig. 4). La vega Armonía corresponde a la producción promedio más alta $4.055 \mathrm{KgMS} \mathrm{ha-}^{-1}$, seguida por Ida Irene con $2.670 \mathrm{KgMS} \mathrm{ha}^{-1}$. Armonía, se caracteriza por la presencia de Acaena magellanica una hierba nativa la cual crece en lugares secos a semi anegados y Agrostis stolonifera, una gramínea que crece muy bien en sitios anegadas permanentemente (Tabla 2). En cambio, Ida Irene es una vega que tiene una composición botánica dominada por especies nativas entre las cuales destaca Carex gayana, una ciperácea que también crece en suelos inundados, junto a Deschampsia antarctica y Hordeum pubiflorum. Al analizar la Figura 5 se infiere que las vegas estudiadas han sido modificadas en su composición botánica, a lo largo del tiempo, debido seguramente la intensidad del pastoreo. En el caso de la vegetación, estas alteraciones se expresan a través de cambios en la biomasa y, en consecuencia, de la productividad primaria neta. 
Tabla 2. Especies que contribuyen a diferenciar la composición florística de los grupos de vegas identificados de acuerdo al análisis SIMPER. Las especies que contribuyen con mayor porcentaje a cada grupo están marcadas en gris.

\begin{tabular}{|c|c|c|c|c|c|}
\hline Especies & $\begin{array}{c}\text { Cobertura } \\
\text { promedio } \\
\text { Grupo } 1\end{array}$ & $\begin{array}{c}\text { Cobertura } \\
\text { promedio } \\
\text { Grupo } 2\end{array}$ & $\begin{array}{c}\text { Cobertura } \\
\text { promedio } \\
\text { Grupo } 3\end{array}$ & $\begin{array}{c}\text { Cobertura } \\
\text { promedio } \\
\text { Grupo } 4\end{array}$ & Origen \\
\hline Acaena magellanica (Lam.) Vahl & 0,444 & 7,56 & 4,67 & 40,4 & Nativa \\
\hline Hordeum pubiflorum Hook.f. subsp. pubiflorum & 13,8 & 21,8 & 13,7 & 7,89 & Nativa \\
\hline Azorella trifurcata (Gaertn.) Pers. & 2,56 & 11,3 & 26,9 & 0 & Nativa \\
\hline Acaena poeppigiana Gay & 0 & 2,59 & 32,9 & 0 & Nativa \\
\hline Carex gayana E. Desv. var. gayana & 21,7 & 1,41 & 0,889 & 1 & Nativa \\
\hline Agrostis stolonifera L. var. stolonifera & 0 & 0,259 & 0 & 23,4 & Introducida \\
\hline Carex acaulis d'Urv. & 4,56 & 12,3 & 3,67 & 0 & Nativa \\
\hline Taraxacum officinale F.H. Wigg. & 1 & 10 & 3,11 & 4,44 & Introducida \\
\hline Deschampsia antarctica E. Desv. & 14,4 & 3,41 & 0,444 & 0 & Nativa \\
\hline Juncus scheuchzerioides Gaudich. & 9 & 6,52 & 0 & 0 & Nativa \\
\hline Poa pratensis L. subsp. pratensis & 7 & 3,56 & 1 & 0,556 & Introducida \\
\hline Caltha sagittata Cav. & 2,67 & 4,48 & 0,889 & 0 & Nativa \\
\hline Leptinella scariosa Cass. & 3,78 & 2 & 0 & 0,222 & Nativa \\
\hline Hordeum lechleri (Steud.) Schenck & 0 & 0 & 0 & 6,89 & Nativa \\
\hline Holcus lanatus L. & 0 & 0 & 0 & 6,22 & Introducida \\
\hline Lobelia oligophylla (Wedd.) Lammers & 2,44 & 2,07 & 0 & 0 & Nativa \\
\hline Deyeuxia poaeoides (Steud.) Rúgolo & 0 & 0 & 0 & 5,44 & Nativa \\
\hline Alopecurus magellanicus Lam. var. magellanicus & 2,33 & 0,222 & 0 & 1,11 & Nativa \\
\hline Colobanthus quitensis (Kunth) Bartl. & 2,67 & 0,444 & 0 & 0 & Nativa \\
\hline Festuca magellanica Lam. & 2,33 & 0,222 & 0,111 & 0 & Nativa \\
\hline Hypochaeris palustris (Phil.) De Wild. & 0 & 1,56 & 0 & 0 & Nativa \\
\hline Gunnera magellanica Lam. & 1,33 & 0,556 & 0 & 0,222 & Nativa \\
\hline Vicia bijuga Gillies ex Hook. E Arn. & 0,556 & 0,926 & 0 & 0 & Nativa \\
\hline Syntrichia sp. (Briófita) & 0 & 0 & 2,11 & 0 & Nativa \\
\hline $\begin{array}{l}\text { Poo spiciformis (Steud.) Hauman \& Parodi var. } \\
\text { spiciformis }\end{array}$ & 0 & 1,07 & 0 & 0 & Nativa \\
\hline Cerastium arvense L. & 0 & 0,111 & 1,78 & 0 & Introducida \\
\hline Azorella fuegiana Speg. & 0 & 0 & 1,78 & 0 & Nativa \\
\hline Colobanthus subulatus (d'Urv.) Hook.f. & 0 & 0 & 1,67 & 0 & Nativa \\
\hline $\begin{array}{l}\text { Gentianella magellanica (Gaudich.) Fabris ex } \\
\text { D.M. Moore }\end{array}$ & 0 & 0,741 & 0,333 & 0 & Nativa \\
\hline Trifolium repens L. & 0 & 0 & 0 & 1,44 & Nativa \\
\hline Indet. (Bríofita) & 1,33 & 0 & 0 & 0 & Nativa \\
\hline $\begin{array}{l}\text { Trisetum spicatum (L.) K. Richt. subsp. cumingii } \\
\text { (Nees ex Steud.) Finot }\end{array}$ & 0 & 0,667 & 0 & 0 & Nativa \\
\hline
\end{tabular}


Nota: Grupo 1: formado por la vega Ida Irene; Grupo 2:

formado por las vegas Punta Catalina, San Miguel, Millaray; Grupo 3: formado por la vega La Frontera y Grupo 4: formado por

la vega Armonía.

Figura 5. Promedio $( \pm \mathrm{DE})$ de la producción de forraje (KgMS $\left.h^{-1}\right)$ de seis vegas, durante 7 meses de evaluación entre los años 2019 y 2020. Letras minúsculas distintas representan diferencias significativas $(p<0,05)$.
Symphyotrichum vahlii (Gaudich.) G.L. Nesom

Arjona pusilla Hook.f.

Cerastium fontanum Baumg.

Perezia pilifera (D. Don) Hook. \& Arn.

Festuca rubra $\mathrm{L}$.

Bromus coloratus Steud.

Poa sp

Ranunculus sp.

Sisyrinchium patagonicum Phil. ex Baker

Eriachaenium magellanicum Sch. Bip.

Hieracium pilosella L. subsp. euronotum Nägeli \& Peter

Gamochaeta nivalis Cabrera

Festuca pyrogea Speg.

Bryum sp. (Briófita)

Primula magellanica Lehm.

Acaena pinnatifida Ruiz \& Pav.

Euphrasia antarctica Benth.

Festuca gracillima Hook.f. var. gracillima

Aira caryophyllea $\mathrm{L}$.

Phleum pratense $\mathrm{L}$.

Stellaria debilis d'Urv.

Contribución total 94

Nro. Total de Especies

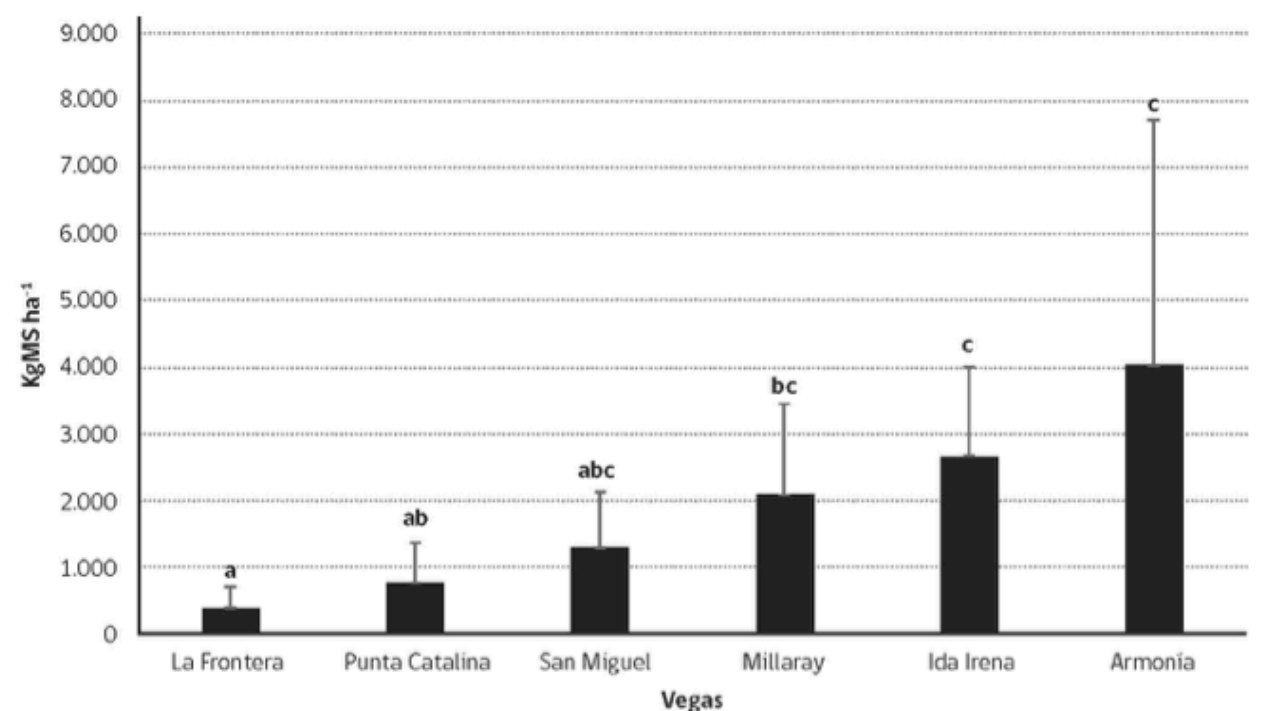


Figura 6. Vista general del grado de infestación de Azorella trifurcata en una vega, en Tierra del Fuego, Región de Magallanes.

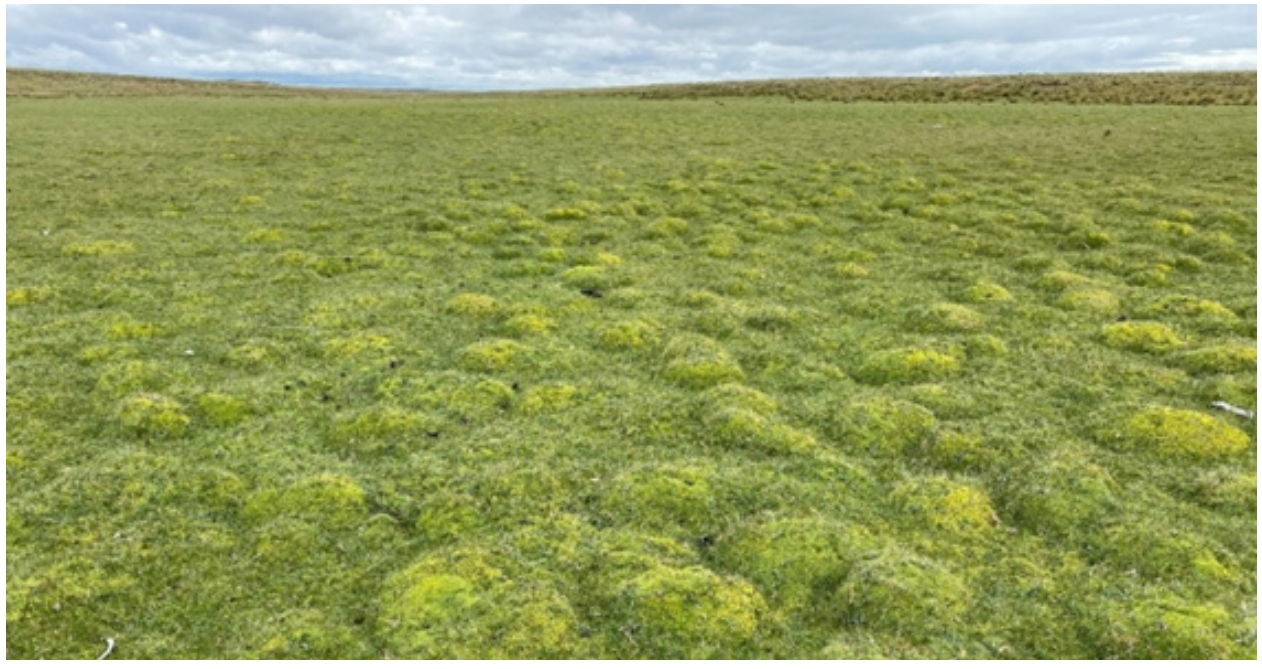

DISCUSIÓN

La información generada en este estudio, muestra que las vegas de Tierra del Fuego no son homogéneas en su composición de especies, ni en su estructura. La vegetación de las vegas estuvo compuesta principalmente por especies pertenecientes a las familias Poaceae, Rosaceae, Cyperaceae, Apiaceae y Asteraceae (Figura 2), lo que no coincide con los resultados obtenidos en estudios realizados en Patagonia Argentina (Boelcke, 1957; Utrilla et al. 2014; Vargas, 2017). En general, las vegas analizadas presentaron valores de riqueza específica e índices de diversidad menores a los encontrados en Patagonia Argentina, específicamente en los sectores de la Provincia de Santa Cruz (Argentina) por Vargas (2017) y los estudios realizados en Magallanes por Filipová et al. (2013) quien registró una riqueza de 37 especies analizando un total de 18 vegas, entre las cuales se incluyeron vegas salinas, que en este estudio no fueron evaluadas.

La composición florística y los atributos de la vegetación mostraron patrones vinculados al contenido hídrico del suelo (Figura 3). Esto coincide con los resultados presentados por Collantes et al. (2013) para Tierra del Fuego y Filipová et al. (2013). Por otra parte, se observa una relación negativa entre el nivel freático y los valores de riqueza e índices de diversidad, es decir a mayor nivel freático, menor riqueza y diversidad (Tabla 1). Respecto a cobertura vegetal, los sectores húmedos presentaron porcentajes de cobertura vegetal mayores a los sectores semihúmedos, ambas relaciones negativa y positiva, coinciden con los estudios realizados por Vargas (2017) y Collantes et al. 2013. Una tendencia similar ha sido descrita para el hemisferio norte por Dwire et al. (2004), quienes explicaron que mientras aumenta la profundidad del nivel freático, se incrementa el número de especies presentes.

Las vegas estudiadas han sido modificadas en su composición botánica, a lo largo del tiempo, debido a los impactos generados por la intensidad del pastoreo. En el caso de la vegetación, estas alteraciones se expresan a través de cambios en la biomasa y, en consecuencia, de la productividad primaria neta (Beschta, 2003; McNaughton, 1984) y cambios en la diversidad de especies (Milchunas $\&$ Lauenroth, 1993; Hickman et al., 2004). La mayor parte de los estudios relacionados con el impacto de la ganadería sobre la estructura y composición se ha desarrollado 
sobre pastizales naturales (coirón) (Oliva et al. 1998). Esto se ajusta a los diversos estudios realizados en Patagonia Argentina (Suárez et al. 2010; Filipová et al. 2013; Utrilla et al. 2014; Grima et al. 2015) los que han reportado los efectos del pastoreo, sobre la estructura de la vegetación, variaciones en la biomasa y la productividad (Suárez et al. 2010) y en la riqueza específica de especies (Ormaechea et al. 2010), en consecuencia, todos estos cambios han generado impactos negativos sobre la productividad, los cuales también pueden ser observados en este estudio en el análisis del PCA (Figura 3).

Por otra parte, el análisis de PCA, se puede interpretar en función de los requerimientos de las especies para los distintos tipos de vegas analizadas, donde el componente 2 representa la disposición de agua en el suelo o nivel freático alto, el cual favorece el crecimiento de Acaena magellanica y Agrostis stolonifera. Por otra parte, la presencia y abundancia de Acaena poeppigea y Azorella trifurcata representa la adaptación de estas para crecer sobre vegas menos anegadas o secas. Siendo Azorella trifurcata una planta nativa indicadora del deterioro de las vegas, lo cual se ajusta a la clasificación propuesta por Suárez et al. (2010); Collantes et al. (2013) y Domínguez et al. (2019). Para Magallanes, esta especie es considerada como una maleza en vegas, la que interfiere con la actividad ganadera (Domínguez, 2020).

Finalmente, algunas recomendaciones prácticas de manejo como medidas para mitigar y mejorar la condición de las vegas estudiadas, serían: 1) Realizar en primavera, un balance forrajero de las vegas, para establecer una carga animal óptima y no usar las vegas en base a una carga fija de animales o manejo histórico, 2) Ajustar la carga animal al balance forrajero, 3) Separar las vegas de los coironales, para evitar el sobrepastoreo de las vegas, 4) Apotrerar las vegas con cerco eléctrico en unidades más pequeñas, acorde con el manejo animal para evitar el sobrepastoreo y su degradación y 5) Disponer de acceso al agua de bebida para los animales en cada potrero dentro de la vega.

Todas estas medidas se justifican, debido a la importancia productiva que tienen las vegas, para la actividad ganadera de Magallanes, las que en promedio representan entre un 20 a $30 \%$ de la superficie predial, pudiendo aportar entre un $40 \%$ y hasta el $50 \%$ del forraje total de la estancia (Pérez et al. 2019).

\section{CONCLUSIONES}

Las condiciones de anegamiento en las vegas limitan la riqueza de especies y la biodiversidad, pero aumenta la productividad, a diferencia de los sectores con menor contenido hídrico, en donde la riqueza específica tiende a aumentar al igual que la biodiversidad, pero disminuye la productividad. Finalmente, para los sitios evaluados en el presente estudio, las condiciones de las vegas están relacionadas a la disponibilidad de agua y su composición botánica, ambas

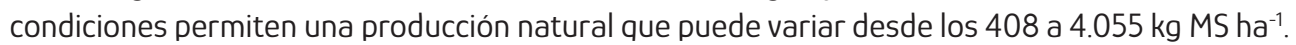

\section{AGRADECIMIENTOS}

Al proyecto: Asesoría para la caracterización predial y elaboración de planes de manejo predial, para 9 unidades productivas ovinas en la provincia de Tierra del Fuego del Programa Territorial Integrado - PTI CORFO. 


\section{LITERATURA CITADA}

Andreazzini, M.J., Degiovanni, S.B., Prieto, A.R., Tripaldi, A., \& Luque, M.E. (2020). Mallines en la Sierra de Comechingones, Sierras Pampeanas, Argentina. Caracterización geológica-geomorfológica \& reconstrucción paleoambiental durante el Holoceno. Andean Geology, 47(1), 77-109.

Beschta, R.L. (2003). Cottonwood, elk, and wolves in the Lamar Valley of Yellowstone National Park. Ecological Applications, 13, 1295 - 1309.

Boelcke, O. (1957). Comunidades herbáceas del Norte de la Patagonia y sus relaciones con la ganadería. Revista de Investigación Agrícola (Argentina), 11, 5-98.

Braun Blanquet, J. (1979). Fitosociología: bases para el estudio de las comunidades vegetales. Blume, Madrid, España.

Clarke, K.R. (1993). Non-parametric multivariate analyses of change in community structure. Australian Journal of Ecology, 18, 117-143.

Collantes, M., Escartín, C., Braun, K., Cingolani, A. \& Anchorena, J. (2013). Grazing and grazing exclusion along a resource gradient in Magellanic meadows of Tierra del Fuego. Rangeland Ecology and Management, 66(6), 688-699.

Correa, MN (1969, 1971, 1978, 1984, 1985, 1988, 1999). Flora Patagónica I-VIII. Colección Científica INTA. Buenos Aires.

Di Rienzo, J., Casanoves, F., Balzarini, M., Gonzalez, L., Tablada, M., \& Robledo, C. (2011). InfoStat versión 2016. Grupo InfoStat, FCA, Universidad Nacional de Córdoba, Argentina. URL http: // www. infostat. com. Arkansas

Domínguez, E., Pérez, C., Suárez, A., Lira, R., Ivelic-Sáez, J., \& de la Fuente, J. (2019). Manual para estimar la cantidad de forraje disponible en pastizales naturales: una herramienta necesaria para un uso racional de estos ecosistemas. Instituto de Investigaciones Agropecuarias. Centro Regional de Investigación Kampenaike. Punta Arenas, Chile. Boletín INIA, 401.

Dupré, C. \& Diekmann, M. (2001). Differences in species richness and life-history traits betweengrazed and abandoned grasslands in southern Sweden. Ecography, 24, 275-286.

Dwire, K., Kauffman, J., Brookshire, E. E Baham, J. (2004). Plant biomass and species composition along an environmental gradient in montane riparian meadows. Oecologia, 139(2), 309-317.

Filipová, L., Hédl, R., \& Covacevich, N. (2010). Variability of soil types in wetland meadows in the south of the chilean Patagonia. Chilean Journal of Agricultural Research, 70(2), 266-277.

Filipová, L., Hédl, R., E Dančák, M. (2013). Magellanic Wetlands: More than Moor. Folia Geobotanica, 48(2), 163-188.

Gaitán, J. J., López, C., \& Bran, D. (2011). Vegetation composition and its relationship with the environment in mallines of north Patagonia, Argentina. Wetlands Ecology and Management, 19, 121-130.

Gaitán, J., Bran, D., Raffo, F., \& Ayesa, J. (2015). Evaluación y cartografía de mallines de las zonas de Loncopué y Chos Malal, provincia del Neuquén. Comunicación técnica, No. 131, área recursos naturales relevamiento integrado. Instituto Nacional de Tecnología Agropecuaria (INTA).

Gandullo, R., \& Schmid, P. (2001). Análisis ecológico de mallines del Parque Provincial Copahue, Neuquén, Argentina. Agro sur, 29(2), 83-99.

Grima, D., Vázquez, M. L., \& Diez, P. G. (2015). Composición florística de pequeñas áreas de mallines con distintas exposición y pendiente. Informes Científicos Técnicos - UNPA, 7(1), 144-161. https://doi. org/10.22305/ict-unpa.v7i1.118

Hammer, Ø., Harper, D. A. T., \& Ryan, P. D. (2001). PAST: Palaeontological statistics software package for education and data analysis. Palaeontologia Electronica, 4(1), 9 pp.

Hickman, K.R., Hartnett, D.C., Cochran, R.C., \& Owensby, C.E. (2004). Grazing management effects on plant species diversity in tallgrass prairie. Journal of Range Management, 57, 58-65.

Pérez, C., Domínguez, E., Ivelic-Sáez, J., Lira, R., Suárez, A., Muñoz, R., Vera, B., \& Sales, F. (2019). Planes de Manejo Predial, "Asesoría para la caracterización predial y elaboración de planes de manejo predial para 9 unidades productivas ovinas en la provincia de Tierra del Fuego, Región de Magallanes y Antártica Chilena", proyecto "Mejoramiento competitivo de la cadena de valor de la lana y la carne ovina en Magallanes" código 16PTI70230-2 CORFO. Informe INIA Kampenaike. 
Mazzoni, E., \& Rabassa, J. (2013). Types and internal hydro-geomorphologic variability of mallines (wet-meadows) of Patagonia: Emphasis on volcanic plateaus. Journal of South American Earth Sciences, 46, 70- 182.

McNaughton, S.J. (1984). Grazing lawns: Animal in herds, plant form, and coevolution. The American Naturalist, 124 , 863-886

Milchunas, D.G., \& Lauenroth, W.K. (1993) Quantitative effects of grazing on vegetation and soils over a global range of environments. Ecological Monographs, 63, 327-366

Oliva, G., Cibils, A., Borrelli, P. \& Humano, G. (1998). Stables states in relation to grazing in Patagonia: a 10-year experimental trial. Journal of Arid Environments, 40, 113- 131.

Ormaechea, S.G., Utrilla, V.R., Suárez, D.D. \& Peri, P.L. (2010). Evaluación objetiva de la condición de mallines de Santa Cruz. Cartilla de Información Técnica. EEA INTA

Santa Cruz. Producción animal, pp 47-52.

Pisano, E. (1977). Fitogeografía de Fuego-Patagonia chilena I. Comunidades vegetales entre las latitudes 52 y $560 S$. Anales del Instituto de la Patagonia, 8, 121-250.

Pykälä, J. (2005). Cattle grazing increases plant species richness of most species trait groups in mesic semi-natural grasslands. Plant Ecology, 175(2), 217-226.

Rodríguez, R. \& Marticorena, A. (Eds.). (2019). Catálogo de las plantas vasculares de Chile. Universidad de Concepción.

Suárez, D., Ormaechea, S.G., Peri, P.L. \& Utrilla, V.R. (2010). Caracterización objetiva de la condición en mallines de Santa Cruz. Inf- técnico. EEA INTA Santa Cruz, 21 pp

Utrilla, V.R., Andrade, M., Billoni, S., Peri, P.L., Sturzenbaum, M.V. \& Rogel, B. (2014). Monitoreo de indicadores de degradación en mallines bajo pastoreo ovino en el Sur de Santa Cruz. EEA INTA Santa Cruz. Informe Técnico Final. 31

Van der Maarel, E. (2007). Transformation of cover-abundance values for appropriate numerical treatment Alternatives to the proposals by Podani. Journal of Vegetation Science, 18, 767-770.

Vargas, P.P. (2017). Mallines del sur de la Patagonia: interacciones entre unidades fisiográficas y productividad en diversos ambientes geomorfológicos. Tesis para obtener el grado de Doctor en Ciencias Aplicadas, presentada en la Universidad Nacional de Luján. 216 pp.

Vázquez, M., Diez, P., \& Grima, D. (2013). Relación suelo. Humedad y composición fisonómica florística del mallín La Carlota. Contribuciones Científicas GAEA, 25, 197-209.

Apéndice: Afiliación declara por cada uno de los autores

\begin{tabular}{|c|c|c|}
\hline $\begin{array}{l}\text { Número } \\
\text { afiliación }\end{array}$ & & Nombre de la inst \\
\hline 1 & \multicolumn{2}{|c|}{ Instituto de Investigaciones Agropecuaria } \\
\hline 2 & \multicolumn{2}{|c|}{ Consultor privado } \\
\hline & Autor & Afiliación \\
\hline \multicolumn{2}{|c|}{ Erwin Domínguez } & 1 \\
\hline \multicolumn{2}{|c|}{ Claudio Pérez } & 2 \\
\hline
\end{tabular}

